The Chinese Society of Theoretical and Applied Mechanics

Chinese Journal of Mechanics Press, Beijing, China

Allerton Press, INC., New York, U.S.A.

\title{
A LATTICE BOLTZMANN METHOD FOR KDV EQUATION*
}

\author{
Yan Guangwu (阎广武 $)^{++++} \quad$ Chen Yaosong (陈耀松 $)^{++} \quad$ Hu Shouxin (胡守信 $)^{+++}$ \\ ${ }^{+}$(Laboratory for Nonlinear Mechanics of Continuous Media, Institute of Mechanics \\ Chinese Academy of Sciences, Beijing 100080, China) \\ ${ }^{++}$(Department of Mechanics, Peking University, Beijing 100871, China) \\ ${ }^{+++}($Department of Mathematics, Jilin University, Changchun 130023, China)
}

\begin{abstract}
We prepose a 5-bit lattice Boltzmann model for KdV equation. Using Chapman-Enskog expansion and multiscale technique, we obtained high order moments of equilibrium distribution function, and the 3rd dispersion coefficient and 4th order viscosity. The parameters of this scheme can be determined by analysing the energy dissipation.
\end{abstract}

KEY WORDS: lattice Boltzmann method, KdV equation, multiscale technique, 5-bit lattice, conservational law in time scale $t_{0}$

\section{INTRODUCTION}

In recent years, the lattice Boltzmann method (LBM) has attracted attention as an alternative numerical scheme for the simulation of fluid flows ${ }^{[1 \sim 3]}$. The main idea of lattice Boltzmann methods are to get available macroscopic physical equations by using the discreted BGK type Boltzmann equation. In general case, time, space and velocity are discreted on one lattice, and then, choose the equilibrium distribution function to fits some requirements which can be obtained with multiscale technique and Chapman-Enskog expansion. Recently, there are some studies about model equations by lattice Boltzmann method ${ }^{[4 \sim 6]}$. On the other hand, equation contained high order partial differential term, such as KdV, can be recovered by modifying the equilibrium distribution function with some high moments, and truncation error of the model controlled to high order.

We expand the distribution function to the third order by Chapman-Enskog expansion, use the conservational law in time scale $t_{0}$, get the error term of model equation of order $O\left(\varepsilon^{3}\right) . \varepsilon$ is Knudsen number.

\section{LATTICE BOLTZMANN MODEL}

\subsection{The Definition of Macroscopic Quantity}

Consider a one dimensional model, we discrede the velocity of particles into four directions, a lattice with unit spacing is used in which each node has four nearest neighbors connected by four links. The distribution function $f_{\alpha}$ is the probability of finding a particle

Received 27 September 1997

* The project supported by the Foundation of the Laboratory for Nonlinear Mechanics of Continuous Media, Institute of Mechanics, Chinese Academy of Sciences 
at time $t$, node $x$, with velocity $e_{\alpha}$, here $\alpha=0,1, \cdots, 4$ ( $\alpha=0$ is rest particle). The particles velocity are $e_{\alpha}=(0, c,-c, k c,-k c), k=2$ are given four neighbors node, see Fig.1. The macroscopic quantity $u(x, t)$ (particles number) was defined by

$$
u(x, t)=\sum_{\alpha} f_{\alpha}(x, t)
$$

The conservational condition was

$$
\sum_{\alpha} f_{\alpha}^{e q}(x, t)=u(x, t)
$$

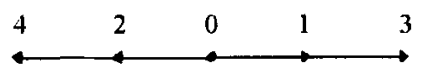

Fig.1 Schematic of a 1D lattice

The particle distribution function satisfy the lattice Boltzmann equations

$$
f_{\alpha}\left(x+e_{\alpha}, t+1\right)-f_{\alpha}(x, t)=-\frac{1}{\tau}\left[f_{\alpha}(x, t)-f_{\alpha}^{e q}(x, t)\right]
$$

where, $f_{\alpha}^{e q}(x, t)$ is the equilibrium distribution function at time $t, x$ and $\tau$ is the single relaxation time factor.

\subsection{A Series of Lattice Boltzmann Equations in Different Time Scales}

Using $\varepsilon$ as the small lattice unit in physical unit, $\varepsilon$ can play the role of the Knudsen number $^{[11]}$, the lattice Boltzmann equation (3) in physical unites is

$$
f_{\alpha}\left(x+\varepsilon e_{\alpha}, t+\varepsilon\right)-f_{\alpha}(x, t)=-\frac{1}{\tau}\left[f_{\alpha}-f_{\alpha}^{e q}\right]
$$

Expanding Eq.(4)

$$
f_{\alpha}\left(x+\varepsilon e_{\alpha}, t+\varepsilon\right)-f_{\alpha}(x, t)=\sum_{n=0}^{\infty} \frac{\varepsilon^{n}}{n !}\left[\frac{\partial}{\partial t}+e_{\alpha} \frac{\partial}{\partial x}\right]^{n} f_{\alpha}(x, t)
$$

and retaining terms up to $O\left(\varepsilon^{5}\right)$ we obtain

$$
\begin{gathered}
\varepsilon\left[\frac{\partial}{\partial t}+e_{\alpha} \frac{\partial}{\partial x}\right] f_{\alpha}+\frac{\varepsilon^{2}}{2}\left[\frac{\partial}{\partial t}+e_{\alpha} \frac{\partial}{\partial x}\right]^{2} f_{\alpha}+\frac{\varepsilon^{3}}{6}\left[\frac{\partial}{\partial t}+e_{\alpha} \frac{\partial}{\partial x}\right]^{3} f_{\alpha}+ \\
\frac{\varepsilon^{4}}{24}\left[\frac{\partial}{\partial t}+e_{\alpha} \frac{\partial}{\partial x}\right]^{4} f_{\alpha}+O\left(\varepsilon^{5}\right)
\end{gathered}
$$

Next, the Chapman-Enskog expansion ${ }^{[7]}$ is applied to $f_{\alpha}$ under the assumption that the mean free path is of the same order of $\varepsilon$. Expand $f_{\alpha}$ anout $f_{\alpha}^{(0)}$

$$
f_{\alpha}=\sum_{n=0}^{\infty} \varepsilon^{n} f_{\alpha}^{(n)}=f_{\alpha}^{(0)}+\varepsilon f_{\alpha}^{(1)}+\varepsilon^{2} f_{\alpha}^{(2)}+\varepsilon^{3} f_{\alpha}^{(3)}+\varepsilon^{4} f_{\alpha}^{(4)}+, \cdots
$$

where, $f_{\alpha}^{e q}$ is $f_{\alpha}^{(0)}$.

To discuss changes in different time scales, we introduce $t_{0}, \cdots, t_{3}$; thus

$$
t_{0}=t, \quad t_{1}=\varepsilon t, \quad t_{2}=\varepsilon^{2} t, \quad t_{3}=\varepsilon^{3} t
$$

and

$$
\frac{\partial}{\partial t}=\frac{\partial}{\partial t_{0}}+\varepsilon \frac{\partial}{\partial t_{1}}+\varepsilon^{2} \frac{\partial}{\partial t_{2}}+\varepsilon^{3} \frac{\partial}{\partial t_{3}}+O\left(\varepsilon^{4}\right)
$$


The equations to the order of $\varepsilon$ is

$$
\frac{\partial f_{\alpha}^{(0)}}{\partial t_{0}}+e_{\alpha} \frac{\partial f_{\alpha}^{(0)}}{\partial x}=-\frac{1}{\tau} f_{\alpha}^{(1)}
$$

The equations to the order of $\varepsilon^{2}$ is

$$
\frac{\partial f_{\alpha}^{(1)}}{\partial t_{0}}+\frac{\partial f_{\alpha}^{(0)}}{\partial t_{1}}+e_{\alpha} \frac{\partial f_{\alpha}^{(1)}}{\partial x}+\frac{1}{2}\left[\frac{\partial}{\partial t_{0}}+e_{\alpha} \frac{\partial}{\partial x}\right]^{2} f_{\alpha}^{(0)}=-\frac{1}{\tau} f_{\alpha}^{(2)}
$$

The equations to the order of $\varepsilon^{3}$ is

$$
\begin{aligned}
& \frac{\partial f_{\alpha}^{(2)}}{\partial t_{0}}+ \frac{\partial f_{\alpha}^{(1)}}{\partial t_{1}}+\frac{\partial f_{\alpha}^{(0)}}{\partial t_{2}}+e_{\alpha} \frac{\partial f_{\alpha}^{(2)}}{\partial x}+\frac{\partial^{2} f_{\alpha}^{(0)}}{\partial t_{0} \partial t_{1}}+e_{\alpha} \frac{\partial^{2} f_{\alpha}^{(0)}}{\partial t_{1} \partial x}+ \\
& \frac{1}{2}\left[\frac{\partial}{\partial t_{0}}+e_{\alpha} \frac{\partial}{\partial x}\right]^{2} f_{\alpha}^{(1)}+\frac{1}{6}\left[\frac{\partial}{\partial t_{0}}+e_{\alpha} \frac{\partial}{\partial x}\right]^{3} f_{\alpha}^{(0)}=-\frac{1}{\tau} f_{\alpha}^{(3)}
\end{aligned}
$$

The equations to the order of $\varepsilon^{4}$ is

$$
\begin{gathered}
\frac{\partial f_{\alpha}^{(0)}}{\partial t_{3}}+\left(\frac{\partial}{\partial t_{0}}+e_{\alpha} \frac{\partial}{\partial x}\right) f_{\alpha}^{(3)}+\frac{\partial f_{\alpha}^{(2)}}{\partial t_{1}}+\frac{\partial f_{\alpha}^{(1)}}{\partial t_{2}}+\frac{1}{24}\left(\frac{\partial}{\partial t_{0}}+e_{\alpha} \frac{\partial}{\partial x}\right)^{4} f_{\alpha}^{(0)}+ \\
\frac{1}{6}\left(\frac{\partial}{\partial t_{0}}+e_{\alpha} \frac{\partial}{\partial x}\right)^{3} f_{\alpha}^{(1)}+\frac{1}{2} \frac{\partial}{\partial t_{1}}\left(\frac{\partial}{\partial t_{0}}+e_{\alpha} \frac{\partial}{\partial x}\right)^{2} f_{\alpha}^{(0)}+\frac{1}{2}\left(\frac{\partial}{\partial t_{0}}+e_{\alpha} \frac{\partial}{\partial x}\right)^{2} f_{\alpha}^{(2)}+ \\
\frac{\partial}{\partial t_{1}}\left(\frac{\partial}{\partial t_{0}}+e_{\alpha} \frac{\partial}{\partial x}\right) f_{\alpha}^{(1)}+\frac{\partial}{\partial t_{2}}\left(\frac{\partial}{\partial t_{0}}+e_{\alpha} \frac{\partial}{\partial x}\right) f_{\alpha}^{(0)}+\frac{1}{2} \frac{\partial^{2} f_{\alpha}^{(0)}}{\partial t_{1}^{2}}=-\frac{1}{\tau} f_{\alpha}^{(4)}
\end{gathered}
$$

From Eq.(9) it follows

$$
\begin{aligned}
& {\left[\frac{\partial}{\partial t_{0}}+e_{\alpha} \frac{\partial}{\partial x}\right]^{2} f_{\alpha}^{(0)}=-\frac{1}{\tau}\left[\frac{\partial}{\partial t_{0}}+e_{\alpha} \frac{\partial}{\partial x}\right] f_{\alpha}^{(1)}} \\
& {\left[\frac{\partial}{\partial t_{0}}+e_{\alpha} \frac{\partial}{\partial x}\right]^{3} f_{\alpha}^{(0)}=-\frac{1}{\tau}\left[\frac{\partial}{\partial t_{0}}+e_{\alpha} \frac{\partial}{\partial x}\right]^{2} f_{\alpha}^{(1)}}
\end{aligned}
$$

Substituting Eq.(10) into Eq.(12), we get

$$
\frac{\partial f_{\alpha}^{(0)}}{\partial t_{1}}-\tau\left(1-\frac{1}{2 \tau}\right)\left(\frac{\partial}{\partial t_{0}}+e_{\alpha} \frac{\partial}{\partial x}\right)^{2} f_{\alpha}^{(0)}=-\frac{1}{\tau} f_{\alpha}^{(2)}
$$

Multiplying by operator $\frac{\partial}{\partial t_{0}}+e_{\alpha} \frac{\partial}{\partial x}$ in Eq.(14),

$$
\left(\frac{\partial}{\partial t_{0}}+e_{\alpha} \frac{\partial}{\partial x}\right) \frac{\partial f_{\alpha}^{(0)}}{\partial t_{1}}+\left(1-\frac{1}{2 \tau}\right)\left(\frac{\partial}{\partial t_{0}}+e_{\alpha} \frac{\partial}{\partial x}\right)^{2} f_{\alpha}^{(1)}=-\frac{1}{\tau}\left(\frac{\partial}{\partial t_{0}}+e_{\alpha} \frac{\partial}{\partial x}\right) f_{\alpha}^{(2)}
$$

and eliminating $f_{\alpha}^{(2)}$ by using Eqs.(11), (11a), (14) and (15), we obtain

$$
\frac{\partial f_{\alpha}^{(0)}}{\partial t_{2}}+(1-2 \tau)\left(\frac{\partial}{\partial t_{0}}+e_{\alpha} \frac{\partial}{\partial x}\right) \frac{\partial f_{\alpha}^{(0)}}{\partial t_{1}}+\left(\tau^{2}-\tau+\frac{1}{6}\right)\left(\frac{\partial}{\partial t_{0}}+e_{\alpha} \frac{\partial}{\partial x}\right)^{3} f_{\alpha}^{(0)}=-\frac{1}{\tau} f_{\alpha}^{(3)}
$$




$$
\begin{aligned}
\frac{\partial f_{\alpha}^{(0)}}{\partial t_{3}}+ & \left(2 \tau^{2}-\frac{5}{2} \tau+\frac{1}{2}\right) \frac{\partial}{\partial t_{1}}\left(\frac{\partial}{\partial t_{0}}+e_{\alpha} \frac{\partial}{\partial x}\right)^{2} f_{\alpha}^{(0)}+ \\
& (1-2 \tau) \frac{\partial}{\partial t_{2}}\left(\frac{\partial}{\partial t_{0}}+e_{\alpha} \frac{\partial}{\partial x}\right) f_{\alpha}^{(0)}+\left(-\tau^{3}+\frac{3}{2} \tau^{2}-\frac{7}{12} \tau+\frac{1}{24}\right) \\
& \left(\frac{\partial}{\partial t_{0}}+e_{\alpha} \frac{\partial}{\partial x}\right)^{4} f_{\alpha}^{(0)}+\frac{\partial f_{\alpha}^{(2)}}{\partial t_{1}}+\frac{1}{2} \frac{\partial^{2} f_{\alpha}^{(0)}}{\partial t_{1}^{2}}=-\frac{1}{\tau} f_{\alpha}^{(4)}
\end{aligned}
$$

Eqs.(9), (14), (16) and (17) satisfy in all dimensional lattice Boltzmann equation generally, it is so-called a series lattice Boltzmann equations in different time scales. The coefficients in Eqs.(14) (17) $\tau-1 / 2, \tau^{2}-\tau+1 / 6$ and $-\tau^{3}+3 \tau^{2} / 2-7 \tau / 12+1 / 24$ are needed in the derivation and may be used to give the feature of macroscopic equations.

\subsection{KdV Equation}

Taking the summation in Eqs.(9), (14), (16) and (17) about $\alpha$, we obtain

$$
\begin{aligned}
& \frac{\partial u}{\partial t_{0}}+\frac{\partial m^{0}}{\partial x}=0 \\
& \frac{\partial u}{\partial t_{1}}+\left(\frac{1}{2}-\tau\right) \sum_{\alpha}\left(\frac{\partial}{\partial t_{0}}+e_{\alpha} \frac{\partial}{\partial x}\right)^{2} f_{\alpha}^{(0)}=0 \\
& \frac{\partial u}{\partial t_{2}}+\left(\tau^{2}-\tau+\frac{1}{6}\right) \sum_{\alpha}\left(\frac{\partial}{\partial t_{0}}+e_{\alpha} \frac{\partial}{\partial x}\right)^{3} f_{\alpha}^{(0)}=0 \\
& \frac{\partial u}{\partial t_{2}}+(1-2 \tau) \sum_{\alpha} \frac{\partial}{\partial t_{2}}\left(\frac{\partial}{\partial t_{0}}+e_{\alpha} \frac{\partial}{\partial x}\right) f_{\alpha}^{(0)}+\left(1-\frac{1}{2 \tau}\right) \sum_{\alpha} \frac{\partial f_{\alpha}^{(2)}}{\partial t_{1}}+ \\
& \quad\left(2 \tau^{2}-2 \tau+\frac{1}{4}\right) \sum_{\alpha} \frac{\partial}{\partial t_{1}}\left(\frac{\partial}{\partial t_{0}}+e_{\alpha} \frac{\partial}{\partial x}\right)^{2} f_{\alpha}^{(0)}+ \\
& \quad\left(-\tau^{3}+\frac{3}{2} \tau^{2}-\frac{7}{12} \tau+\frac{1}{24}\right) \sum_{\alpha}\left(\frac{\partial}{\partial t_{0}}+e_{\alpha} \frac{\partial}{\partial x}\right)^{4} f_{\alpha}^{(0)}=0
\end{aligned}
$$

$(18)+(19) \times \varepsilon+(20) \times \varepsilon^{2}+(21) \times \varepsilon^{3}$ results in

$$
\begin{gathered}
\frac{\partial u}{\partial t}+\frac{\partial m^{0}}{\partial x}+\varepsilon\left(\frac{1}{2}-\tau\right) \sum_{\alpha}\left(\frac{\partial}{\partial t_{0}}+e_{\alpha} \frac{\partial}{\partial x}\right)^{2} f_{\alpha}^{(0)}+\varepsilon^{2} \mu \sum_{\alpha}\left(\frac{\partial}{\partial t_{0}}+e_{\alpha} \frac{\partial}{\partial x}\right)^{3} f_{\alpha}^{(0)}+ \\
\varepsilon^{3}\left[\frac{\partial u}{\partial t_{2}}+(1-2 \tau) \sum_{\alpha} \frac{\partial}{\partial t_{2}}\left(\frac{\partial}{\partial t_{0}}+e_{\alpha} \frac{\partial}{\partial x}\right) f_{\alpha}^{(0)}+\left(1-\frac{1}{2 \tau}\right) \sum_{\alpha} \frac{\partial f_{\alpha}^{(2)}}{\partial t_{1}}+\right. \\
\left(2 \tau^{2}-2 \tau+\frac{1}{4}\right) \sum_{\alpha} \frac{\partial}{\partial t_{1}}\left(\frac{\partial}{\partial t_{0}}+e_{\alpha} \frac{\partial}{\partial x}\right)^{2} f_{\alpha}^{(0)}+ \\
\left.\left(-\tau^{3}+\frac{3}{2} \tau^{2}-\frac{7}{12} \tau+\frac{1}{24}\right) \sum_{\alpha}\left(\frac{\partial}{\partial t_{0}}+e_{\alpha} \frac{\partial}{\partial x}\right)^{4} f_{\alpha}^{(0)}\right]=0
\end{gathered}
$$

Under the assumption that

$$
\begin{aligned}
& m^{0}=\sum_{\alpha} f_{\alpha}^{(0)} e_{\alpha}=\frac{1}{2} u^{2} a \\
& \pi^{0}=\sum_{\alpha} f_{\alpha}^{(0)} e_{\alpha}^{2}=\frac{1}{3} u^{3} a^{2}
\end{aligned}
$$


where $a$ is constant number, we have

$$
\sum_{\alpha}\left(\frac{\partial}{\partial t_{0}}+e_{\alpha} \frac{\partial}{\partial x}\right) f_{\alpha}^{(0)}=0 \quad \sum_{\alpha}\left(\frac{\partial}{\partial t_{0}}+e_{\alpha} \frac{\partial}{\partial x}\right)^{2} f_{\alpha}^{(0)}=0
$$

In fact, Eq.(25) is the conservational law of $u$ and $m^{0}$, this is so-called conservational law in time scale $t_{0}$. It plays an important role in the construction of lattice Boltzmann scheme of high order precision. Equation (25) had also been shown in references [5] and [6], but those are not conservational law in time scale $t_{0}$.

Equation (22) become

$$
\frac{\partial u}{\partial t}+\frac{\partial}{\partial x}\left(\frac{1}{2} a u^{2}\right)+\varepsilon^{2} \mu \sum_{\alpha}\left(\frac{\partial}{\partial t_{0}}+e_{\alpha} \frac{\partial}{\partial x}\right)^{3} f_{\alpha}^{(0)}+\varepsilon^{3} \eta \sum_{\alpha}\left(\frac{\partial}{\partial t_{0}}+e_{\alpha} \frac{\partial}{\partial x}\right)^{4} f_{\alpha}^{(0)}=0
$$

where $\mu=\tau^{2}-\tau+1 / 6, \eta=\left(-\tau^{3}+\frac{3}{2} \tau^{2}-\frac{7}{12} \tau+\frac{1}{24}\right)$. The third term of Eq.(26)'s left hand side is

$$
\varepsilon^{2} \mu \sum_{\alpha}\left(\frac{\partial}{\partial t_{0}}+e_{\alpha} \frac{\partial}{\partial x}\right)^{3} f_{\alpha}^{(0)}=\varepsilon^{2} \mu \frac{\partial^{2}}{\partial x^{2}}\left[\frac{\partial \pi^{0}}{\partial t_{0}}+\frac{\partial P^{0}}{\partial x}\right]
$$

where $P^{0}=\sum_{\alpha} f_{\alpha}^{(0)} e_{\alpha}^{3}$. Denote $L^{0}=\sum_{\alpha} f_{\alpha}^{(0)} e_{\alpha}^{4}$, and choose

$$
P^{0}=\xi_{1} u+a^{3} \frac{1}{4} u^{4} \quad L^{0}=\xi_{2} \frac{1}{2} a u^{2}+a^{4} \frac{1}{5} u^{5}
$$

thus

$$
\begin{aligned}
& \frac{\partial \pi^{0}}{\partial t_{0}}+\frac{\partial P^{0}}{\partial x}=\xi_{1} \frac{\partial u}{\partial x} \\
& \frac{\partial P^{0}}{\partial t_{0}}+\frac{\partial L^{0}}{\partial x}=\xi_{2} \frac{\partial\left(\frac{1}{2} a u^{2}\right)}{\partial x}
\end{aligned}
$$

Equation (26) becomes $\mathrm{KdV}$ equation ${ }^{[8 \sim 10]}$, therefore

$$
\frac{\partial u}{\partial t}+\frac{\partial}{\partial x}\left(\frac{1}{2} a u^{2}\right)+\nu \frac{\partial^{3} u}{\partial x^{3}}=O\left(\varepsilon^{3}\right)
$$

The truncation error is

$$
R=O\left(\varepsilon^{3}\right)=\lambda \frac{\partial^{4}}{\partial x^{4}}\left(\frac{1}{2} a u^{2}\right)+O\left(\varepsilon^{4}\right)
$$

Here the coefficent $\nu$ is given as

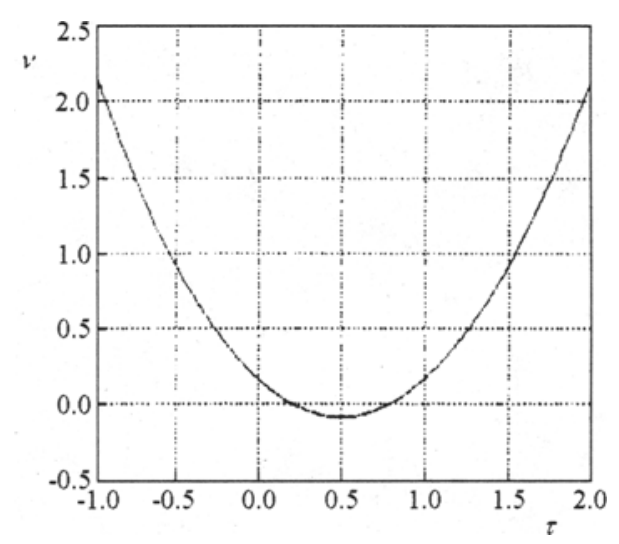

$$
\nu=\xi_{1} \varepsilon^{2} \mu=\varepsilon^{2}\left(\tau^{2}-\tau+\frac{1}{6}\right) \xi_{1}
$$

Fig.2 The curve of $\frac{\nu}{\xi_{1} \varepsilon^{2}}$ versus $\tau$

From Eq.(32), the parameters $\xi_{1}, \nu$ and $\varepsilon$ can be used to determine the relaxation factor $\tau$, and $\xi_{1}$ is chosen to satisfy

$$
\frac{1}{12}+\frac{\nu}{\varepsilon^{2} \xi_{1}}>0
$$


The 4 th viscosity is given by

$$
\lambda=\varepsilon^{3}\left(\tau^{3}-\frac{3}{2} \tau^{2}+\frac{7}{12} \tau-\frac{1}{24}\right)\left(4 \xi_{1}-\xi_{2}\right)
$$

and then

$$
\lambda=\varepsilon^{3}\left(\tau-\frac{1}{2}\right)\left(\tau^{2}-\tau+\frac{1}{12}\right)\left(4 \xi_{1}-\xi_{2}\right)
$$

Equation (34) is written as

$$
\lambda=\varepsilon\left(\frac{\nu}{\xi_{1}}-\frac{1}{12} \varepsilon^{2}\right) \sqrt{\frac{1}{12}+\frac{\nu}{\varepsilon^{2} \xi_{1}}}\left(4 \xi_{1}-\xi_{2}\right)
$$

where $\nu=1 / \sigma^{2}$. Choosing $\lambda>0$, we get

$$
\varepsilon \sigma<\sqrt{\frac{12}{\xi_{1}}}
$$

It is an important conclusion that Eq.(36) become stability criterion for lattice Boltzmann equation. The parameters $\xi_{1}$ and $\xi_{2}$ are given by the model energy dissipation.

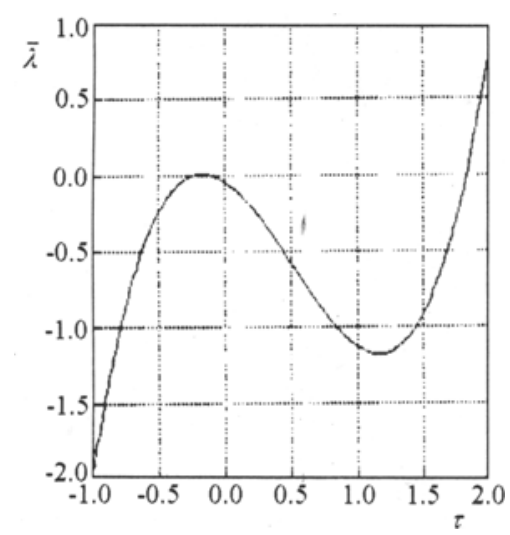

Fig.3 The curve of the 4 th viscosity $\bar{\lambda}=\frac{\lambda\left(4 \xi_{1}-\xi_{2}\right)}{\varepsilon^{3}}$ versus $\tau$

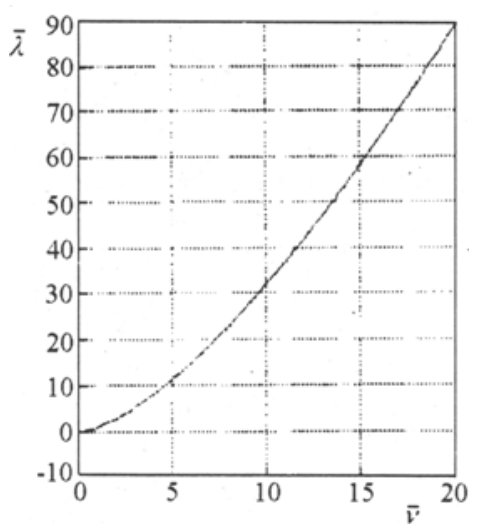

Fig.4 The relationship between the 3rd dispersion coefficent $\bar{\nu}=\frac{\nu}{\xi_{1} \varepsilon^{2}}$ and $\lambda$

\subsection{The Energy Dissipation of the Lattice Boltzmann Model}

Assuming that particles satisfy energy conservation in the form

$$
\begin{gathered}
m=\sum_{\alpha} f_{\alpha} e_{\alpha}=\sum_{\alpha} f_{\alpha}^{(0)} e_{\alpha}=m^{0} \\
\sum_{\alpha} f_{\alpha}^{(k)} e_{\alpha}=0 \quad k \geq 1
\end{gathered}
$$

Equations (9), (14) and (16) multiplying by $e_{\alpha}$ and taking the summation we get

$$
\begin{aligned}
& \frac{\partial m}{\partial t_{0}}+\frac{\partial \pi^{0}}{\partial x}=0 \\
& \frac{\partial m}{\partial t_{1}}+\left(\frac{1}{2}-\tau\right) \xi_{1} \frac{\partial^{2} u}{\partial x^{2}}=0
\end{aligned}
$$




$$
\frac{\partial m}{\partial t_{2}}+\left(\tau^{2}-\tau+\frac{1}{6}\right)\left(\xi_{1}-3 \xi_{2}\right) \frac{\partial^{3}}{\partial x^{3}}\left(\frac{1}{2} a u^{2}\right)=0
$$

Back to the time scale $t$, we have

$$
\frac{\partial m}{\partial t}+\frac{\partial \pi^{0}}{\partial x}+\varepsilon \xi_{1}\left(\frac{1}{2}-\tau\right) \frac{\partial^{2} u}{\partial x^{2}}+\varepsilon^{2}\left(\xi_{2}-3 \xi_{1}\right)\left(\tau^{2}-\tau+\frac{1}{6}\right) \frac{\partial^{3}}{\partial x^{3}}\left(\frac{1}{2} u^{2}\right)+O\left(\varepsilon^{3}\right)=0
$$

Here, the remaining coefficients are determined by the positive condition of Eq.(41).

\subsection{The Local Equilibrium Distribution}

The moments of $f_{\alpha}^{(0)}$ can be expressed as

$$
\begin{aligned}
& \sum_{\alpha} f_{\alpha}^{(0)}=u=B_{1} \\
& \sum_{\alpha} f_{\alpha}^{(0)} e_{\alpha}=\frac{1}{2} u^{2} a=c B_{2} \\
& \sum_{\alpha} f_{\alpha}^{(0)} e_{\alpha}^{2}=\frac{1}{3} u^{3} a^{2}=c^{2} B_{3} \\
& \sum_{\alpha} f_{\alpha}^{(0)} e_{\alpha}^{3}=\frac{1}{4} u^{4} a^{3}+\xi_{1} u=c^{3} B_{4} \\
& \sum_{\alpha} f_{\alpha}^{(0)} e_{\alpha}^{4}=\frac{1}{5} u^{5} a^{4}+\xi_{2} \frac{1}{2} u^{2} a=c^{4} B_{5}
\end{aligned}
$$

We get the equilibrium distribution as

$$
\begin{aligned}
f_{1}^{(0)} & =\frac{1}{2} \frac{k^{2} B_{3}-B_{5}+k^{2} B_{2}-B_{4}}{k^{2}-1} \\
f_{2}^{(0)} & =\frac{1}{2} \frac{k^{2} B_{3}-B_{5}-k^{2} B_{2}+B_{4}}{k^{2}-1} \\
f_{3}^{(0)} & =\frac{1}{2}\left(\frac{B_{5}-B_{3}}{k^{4}-k^{2}}+\frac{B_{4}-B_{2}}{k^{3}-k}\right) \\
f_{4}^{(0)} & =\frac{1}{2}\left(\frac{B_{5}-B_{3}}{k^{4}-k^{2}}-\frac{B_{4}-B_{2}}{k^{3}-k}\right) \\
f_{0}^{(0)} & =B_{1}-\left(f_{1}^{(0)}+f_{2}^{(0)}+f_{3}^{(0)}+f_{4}^{(0)}\right)
\end{aligned}
$$

\section{NUMERICAL EXAMPLE}

A test problem, the collison of two solitons ${ }^{[10]}$, with initial and boundary function

$$
\begin{aligned}
& \frac{\partial u}{\partial t}+u \frac{\partial u}{\partial x}+\mu \frac{\partial^{3} u}{\partial x^{3}}=0 \\
& -\infty \leq x \leq \infty \quad 0<t<T \\
& u(x, 0)=3 c_{1} \operatorname{sech}^{2}\left(k_{1} x+d_{1}\right)+3 c_{2} \operatorname{sech}^{2}\left(k_{2} x+d_{2}\right) \\
& c_{1}=0.3 \quad c_{2}=0.1 \quad d_{1}=-6.0 \quad d_{2}=-6.0 \\
& k_{1}=\frac{1}{2}\left(c_{1} / \mu\right)^{1 / 2} \quad k_{2}=\frac{1}{2}\left(c_{2} / \mu\right)^{1 / 2}
\end{aligned}
$$


was simulated by using this model. It shows that bigger soliton should reach and collide with smaller soliton in the process. For the phenomenom of swallowing and spitting, see Fig.5. In the process of the collision, all particles number is conserved, see Fig.6, but the total particles energy has a bit dissipation see Fig.7. The Fig.5 shows the process of swallowing and spitting when two solitons collide.
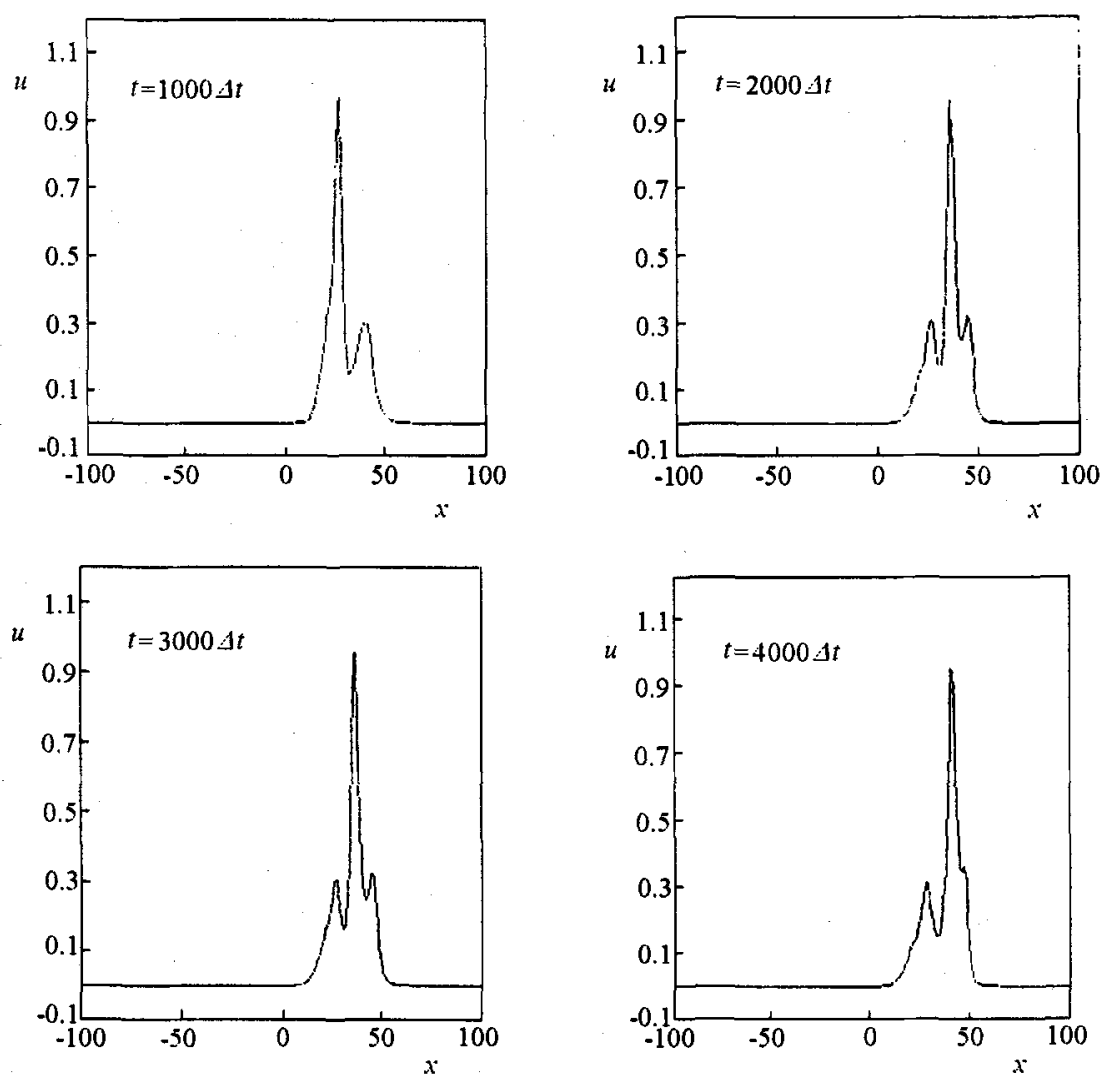

Fig.5 The process of two solitons collision, $c=10.0, \mu=1.0$
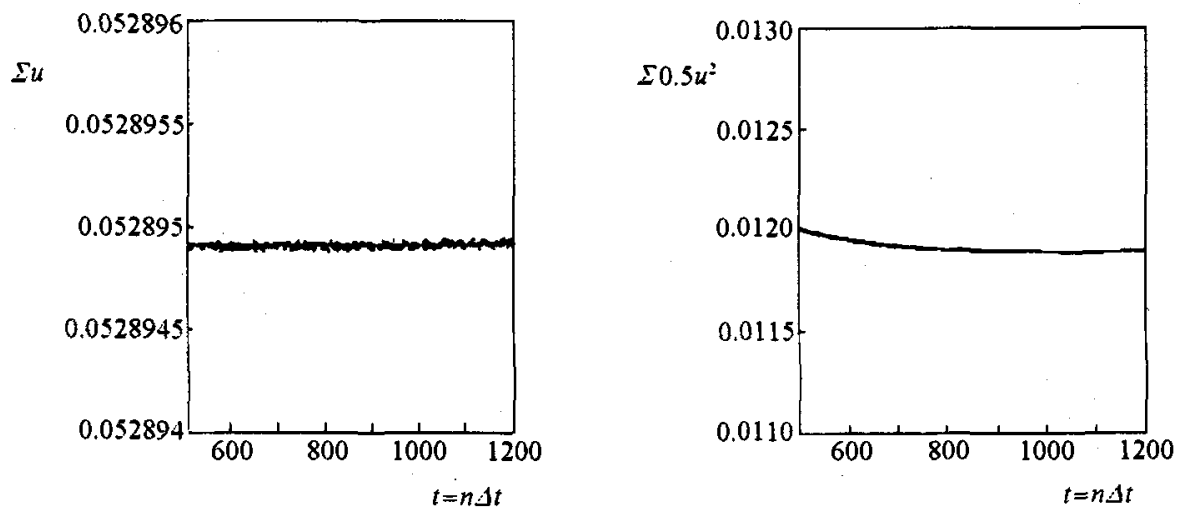

Fig.6 All particles number

Fig.7 All particles energy, $c=3.0, \mu=1.0$ 


\section{CONCLUSION}

There was a famous method, Grad-13 moments equations in the area of gas dynamics, which was successful in the simulation of Navier-Stokes equations from Boltzmann equation. In 1991, U. Frisch pointed out the possibility that the requirments of higher moments may be used to construct lattice gas model for Navier-Stokes equation ${ }^{[12]}$. In this paper, we carried out Chapman-Enskog expanson and multiscale technique on the distribution function, obtained $f_{\alpha}^{(3)}$, and KdV equation with high order accuracy. The conservational law in time scale $t_{0}$, the equations of different time scales Eqs.(9), (14), (16) and (17) are important results in the lattice Boltzmann method.

The lattice Boltzmann model for KdV equation is simple, but Euler equations and Navier-Stokes are more complex which is the next step of the authors.

Acknowledgements: The authors are grateful to Dr Liu Riwu and Dr Xiong Shengwu for helpful discussions.

\section{REFERENCES}

1 Qian YH, d'humieres D, Lallemand P. Lattice BGK Model for Navier-Stokes Equation. Europhys Lett, 1992, 17(6): 479 484

2 Chen HD, Chen SY, Matthaeus MH. Recovery of the Navier-Stokes Equations Using a Lattice Boltzmann Method. Phys Rev, 1992, 45A: 5339 5342

3 Benzi R, Succi S, Vergassola M. The Lattice Boltzmann Equations: Theory and Applications. Physics Reports, 1992, 222: 147 197

4 Alexander FJ, Chen HD, Chen SY, Doolen GD. Lattice Boltzmann Model for Compressible Fluids. Phys Rev, 1993, 46A: 1967 1970

5 Zou Xiufen. A lattice model for convection diffusion equation. Chinese Journal of Computational Physics, 1996, 3: 310 314

6 Yan GW, Hu SX, Shi WP. A difference type lattice gas scheme for conservational equation. Chinese Journal of Computational Physics, 1997, 2: 190 194

7 Chapman S, Cowling TG. The Mathematical Theory Non-Uniform Gas. Cambridge: Cambridge University Press, 1939

8 Whitham GB. Linear and Nonlinear Waves. New York: A Wiley-Interscience Publication, 1974

9 Guo BL, Pang XF. Soliton, Beijing: Science Press, 1987

10 Xin XK, Liu RX, Jiang BC. Computational Fluid Dynamics. Changsha, China: National University of Defence Technology Press, 1989

11 Hou SL, Zhou QS, Chen SY, et al. Simulation of cavity flow by the lattice Boltzmann method. $J$ Comput Phys, 1995, 118: 329 347

12 Frisch U. Relation between the lattice Boltzmann equation and the Navier-Stokes equation. Physica, 1991, 47D: 231 232 